\title{
Interaction of Botulinum Toxin with the Epithelial Barrier
}

\author{
Yukako Fujinaga \\ Laboratory for Infection Cell Biology, International Research Center for Infectious Diseases, Research Institute for Microbial Diseases, \\ Osaka University, Yamada-oka 3-1, Suita, Osaka 565-0871, Japan
}

Correspondence should be addressed to Yukako Fujinaga, yukafuji@biken.osaka-u.ac.jp

Received 10 August 2009; Accepted 24 December 2009

Academic Editor: Karl Chai

Copyright (c) 2010 Yukako Fujinaga. This is an open access article distributed under the Creative Commons Attribution License, which permits unrestricted use, distribution, and reproduction in any medium, provided the original work is properly cited.

\begin{abstract}
Botulinum neurotoxin $(\mathrm{BoNT})$ is a protein toxin $(\sim 150 \mathrm{kDa})$, which possesses a metalloprotease activity. Food-borne botulism is manifested when BoNT is absorbed from the digestive tract to the blood stream and enters the peripheral nerves, where the toxin cleaves core proteins of the neuroexocytosis apparatus and elicits the inhibition of neurotransmitter release. The initial obstacle to orally ingested BoNT entering the body is the epithelial barrier of the digestive tract. Recent cell biology and molecular biology studies are beginning to elucidate the mechanism by which this large protein toxin crosses the epithelial barrier. In this review, we provide an overview of the structural features of botulinum toxins (BoNT and BoNT complex) and the interaction of these toxins with the epithelial barrier.
\end{abstract}

\section{Introduction}

The botulinum neurotoxin (BoNT, Mr. $\sim 150 \mathrm{kDa}$, types A to $G)$, which is produced by various strains of the anaerobic spore-forming bacteria Clostridium botulinum, C. butyricum, and $C$. baratii, is one of the most toxic proteins to humans. BoNT is the etiologic agent that causes botulism, a severe neurological disease characterized by flaccid paralysis. The most common mechanism of botulism poisoning is through oral ingestion of the toxin contaminated in food. The lethal dose of BoNT for humans is not known but has been estimated in primate studies; the estimated lethal amount of crystalline type A toxin (main ingredient is 19S toxin, see chapter II-1) for a $70 \mathrm{~kg}$ human is approximately 0.09 $0.15 \mathrm{ug}$ intravenously or intramuscularly, and $70 \mathrm{ug}$ orally (reviewed in [1]). BoNT binds specifically to neuronal cells, enters the cytoplasm, and then cleaves the core proteins involved in the vesicular fusion machinery by its metalloprotease activity, thereby blocking the release of neurotransmitters (reviewed in [2]). When produced by the bacterium, the BoNT is found in complexes (BoNT complexes, progenitor toxins) associated with nontoxic components (reviewed in [3-7], Figures 1(a) and 2).

To cause disease, orally ingested BoNT in the complexes must take a long journey to reach their targets, the peripheral nerves (Figure 1(b)). The initial obstacle to orally ingested
BoNT entering the body is the epithelial barrier of the digestive tract. Although the molecular mechanism by which this large protein toxin crosses the epithelial barrier is not completely defined, recent studies have led to a progressive understanding of the interaction of BoNT and BoNT complexes with the epithelial barrier.

Here, we describe the structure-activity relationship of botulinum toxins (BoNT and BoNT complexes) and cover recent advances in our understanding of the transport pathway followed by these toxins from the gut lumen to the general circulation across the epithelial barrier.

\section{Structural Aspects of Botulinum Neurotoxin Complex}

2.1. Overview of Molecular Composition of BoNT Complex. Botulinum neurotoxin (BoNT/NTX/7S toxin) is classified into seven serotypes, BoNT/A through BoNT/G, on the basis of their immunological properties. In addition, the variation observed in BoNT protein sequences within the serotypes, at least in serotype $\mathrm{A}-\mathrm{F}$, has resulted in designations of BoNT subtypes within a serotype [11-15]. For example, five subtypes of BoNT/A (termed A1-A5) have been identified. Subtypes are defined as differing by at least $2.6 \%$ at the amino acid level $[11,12]$. These subtypes BoNT are produced by the bacterium as complexes (BoNT complexes/progenitor 


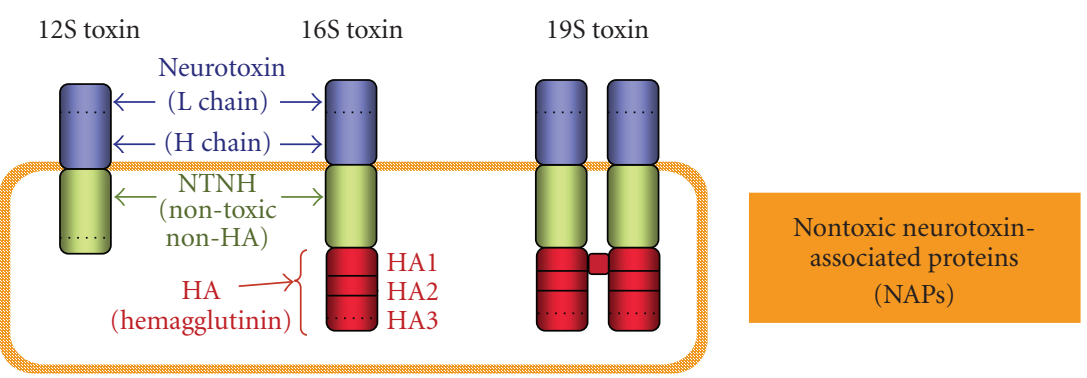

(a) BoNT complex

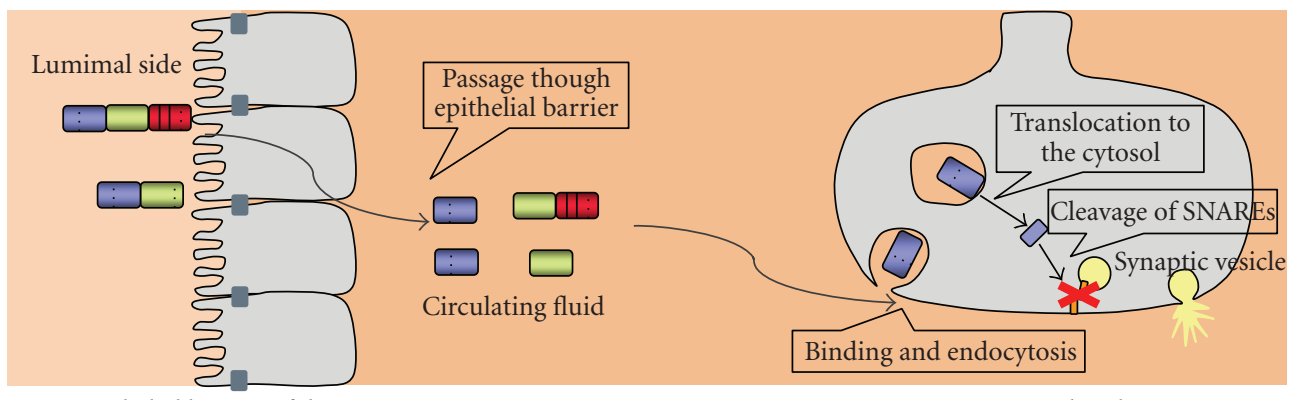

Epithelial barrier of digestive tract

Peripheral nerves

(b) Food-borne botulism

FIGURE 1: Botulinum neurotoxin complexes and food-borne botulism. (a) Schematic structure of botulinum neurotoxin (BoNT) complexes. (b) The pathway followed by BoNT complexes from the lumen of the intestinal tract to the cytosol of the peripheral nerve terminal in foodborne botulism. Orally ingested BoNT complexes (12S and $16 \mathrm{~S}$ toxins) must cross the intestinal epithelial barrier to cause the food-borne botulism. After absorption from the small intestine, the botulinum neurotoxin complexes enter the lymphatic system, then the blood stream $[3,8]$. In the lymphatic circulation and blood, BoNT exists as a free form dissociated from the complex [3,9] and binds specifically to neurons [2, 10]. Inhibition of neurotransmitter release occurs via a four-step mechanism, (1) binding, (2) endocytosis, (3) translocation, and (4) cleavage of the SNARE proteins [2].

\begin{tabular}{llc}
\hline \multirow{2}{*}{ Type } & \multicolumn{3}{c}{ BoNT complex } \\
\cline { 2 - 3 } & HA(+) complex & HA(-) complex \\
\hline & 16S toxin & 19 S toxin \\
B & & \\
C & & \\
D & & \\
E & & \\
F & & \\
G
\end{tabular}

$\square$ BoNT $\square$ HANH

Figure 2: Features of types A to G BoNT complexes. Type A (A1) BoNT is produced by C. botulinum in three forms: 12S, 16S, and 19S toxins. Types B, C, and D BoNT are produced in two forms: $16 \mathrm{~S}$ and $12 \mathrm{~S}$ toxins. Types E, F, (and A2) BoNT are produced as $12 \mathrm{~S}$ toxin. Type G BoNT complex is produced as $16 \mathrm{~S}$ toxin (reviewed in $[3,6,7]$ ).

toxins) associated with nontoxic components (nontoxic neurotoxin-associated proteins, NAP) (reviewed in [3-7] Figures 1(a) and 2). Three forms of BoNT complexes, $12 \mathrm{~S}$ toxin (M toxin/M-TC), 16S toxin (L toxin/L-TC), and 19S toxin (LL toxin/LL-TC), are the major forms in cultures of the bacteria. $12 \mathrm{~S}$ toxin is composed of a BoNT and a nontoxic nonhemagglutinin (non-toxic non-HA, NTNH, also called as NTNHA; $130 \mathrm{kDa}$ ). $16 \mathrm{~S}$ toxin is composed of a BoNT, an NTNH, and several hemagglutinin (HA) proteins. 19S toxin has the same components as $16 \mathrm{~S}$ toxin and is presumed to be 
a dimer of two $16 \mathrm{~S}$ toxins linked by one of the HA proteins [16]. C. botulinum type A (A1) strain produces $12 \mathrm{~S}, 16 \mathrm{~S}$, and $19 \mathrm{~S}$ toxins. Type B, C, and D strains produce $16 \mathrm{~S}$ and $12 \mathrm{~S}$ toxins. Type A2, E, and F strains only produce $12 S$ toxin. Type G strain produces only $16 \mathrm{~S}$ toxin (reviewed in $[3,6,7]$ ). Toxin types $\mathrm{A}, \mathrm{B}, \mathrm{E}$, and $\mathrm{F}$ cause botulism in both humans and animals, whereas types $C$ and $D$ cause botulism mainly in animals, but very rarely in humans. Type $\mathrm{G}$ toxin producing organisms have been experimentally isolated from soil, but no naturally occurring outbreaks of botulism caused by type $\mathrm{G}$ toxin have been reported (reviewed in $[1,7]$ ).

In this review, when it is not suitable to use the precise terms, BoNT and BoNT complex (i.e., BoNT plus NAP), we will use the term "botulinum toxin" which means either BoNT or BoNT complex.

2.2. Structure and Activity of BoNT. BoNT is synthesized as single-polypeptide chains of $\sim 150 \mathrm{kDa}$ but must be posttranslationally modified by a bacterial or tissue protease for activation (reviewed in [2]). The active form of the toxin consists of a light chain $(\mathrm{L}, 50 \mathrm{kDa})$ and a heavy chain $(\mathrm{H}, 100 \mathrm{kDa})$. The chains remain covalently and reversibly linked by a disulphide bond until exposed to reducing conditions, such as in the nerve cytosol. The L chain is a zinc endopeptidase, which cleaves the SNARE proteins VAMP (vesicle-associated membrane protein/synaptobrevin; cleaved by BoNT/B, D, F, and G), which is located in SV (synaptic vesicles), and SNAP-25 (25 kDa synaptosome associated proteins; cleaved by BoNT types A, C, and E) and syntaxin (cleaved by BoNT/C), which are expressed mainly in the plasma membrane [2]. The $\mathrm{H}$ chain is composed of two domains and serves as the vehicle that delivers the $\mathrm{L}$ chain into the cytosol of neuronal cells. The carboxyterminal part of the $\mathrm{H}$ chain $(\mathrm{Hc})$ is mainly responsible for neurospecific binding via its interaction with specific gangliosides and protein receptors (dual-receptor model, reviewed in $[2,10])$. Regarding protein receptors, synaptic vesicle protein 2 (SV2) has been proposed to be a protein receptor for BoNT/A (all three isoforms SV2A, B and C [17], SV2C [18]), BoNT/E (SV2A and B [19]) and BoNT/F (SV2A, B and C [20]), while synaptotagmin I and II (SytI an -II) have been identified as protein receptors for both BoNT/B and BoNT/G [21-27]). In contrast, BoNT/C and BoNT/D seem to only interact with gangliosides (GD1b and GT1b) and phosphatidylethanolamine, respectively [28]. The aminoterminal part of the $\mathrm{H}$ chain $\left(\mathrm{H}_{\mathrm{N}}\right)$ is thought to mediate translocation of the $\mathrm{L}$ chain from the lumen of an acidic intracellular compartment into the cytosol [29-31].

Information from the crystallographic structures of BoNT [32-37], cocrystallographic structures of BoNT with their SNARE substrates [38-40], and cocrystallographic structures of BoNT with receptors [41-44] have revealed the details of these interactions at the atomic level.

2.3. NTNH and HA Proteins. The nontoxic components (NAP) of $12 \mathrm{~S}$ and $16 \mathrm{~S}$ toxins are composed of an NTNH and an NTNH with several HA proteins, respectively. All these components and a BoNT are associated with each other by noncovalent binding. The BoNT dissociates from
NAP in slightly alkaline conditions (higher than $\mathrm{pH} 7.2 \sim 8$ ) (reviewed in [3]).

NTNHs are produced by all types (strains) of $C$. botulinum and are the most conserved proteins in the botulinum neurotoxin complex (their amino acid identity level is 76 83.5\%) (reviewed in [5]). The biological and structural roles of NTNH are not completely understood, although it is believed that they confer resistance to proteolysis in the gastrointestinal tract (reviewed in [3], for type D NTNH [45]).

The HA component consists of three different proteins: HA1 (also referred to as HA-33 in types C and D, HA-34 in type $\mathrm{B}$, and HA-35 in type A, based on their molecular weight), HA2 (also referred to as HA-15 in type A, HA-17 in types $\mathrm{C}$ and $\mathrm{D}$, and HA-18 in type B), and HA3 (also referred to as HA-70, a precursor form of HA3a and HA3b) [6]).

The $12 \mathrm{~S}$ toxin is composed of a BoNT and an NTNH at a $1: 1$ ratio (reviewed in $[3,6,45]$ ). $16 \mathrm{~S}$ toxin results from the assembly of a BoNT, an NTNH, and HA proteins (HA1, HA2, and HA3) at a presumed $1: 1: 6: 3: 3[46]$ or $1: 1: 4: 4: 2$ ratio $[47,48]$.

The X-ray crystallographic structures of HA1 (type C $[49,50]$, type A [51]), HA1-HA2 complex (type D [46]), and HA3 (type C [52]) have been determined. Furthermore, recent transmission electron microscopy studies of type D $16 \mathrm{~S}$ toxin suggest an ellipsoidal-shaped structure with 3 extended arms [46]. Other such studies will provide valuable information for understand the precise three- and fourdimensional structures of $12 \mathrm{~S}$ and $16 \mathrm{~S}$ toxin, although the crystallographic structures of these BoNT complexes have not yet been elucidated.

\section{Mechanism of the Passage of Botulinum Toxins through the Digestive Tract}

3.1. Site of Absorption of Botulinum Toxins. Botulinum toxin cannot penetrate intact skin, but the toxin is absorbed from mucosal surfaces or a wound (reviewed in [1]). In foodborne botulism and in intestinal botulism (infant and adult, botulinum toxins are produced from C. botulinum and other BoNT producing clostridia, which colonize the lumen of intestine), the toxins are absorbed from the digestive tract. During naturally occurring food-borne botulism, abdominal cramp, nausea, vomiting, or diarrhea are often reported. These gastrointestinal symptoms are thought to be caused by other bacterial metabolites and may not occur if pure botulinum toxins are contaminated in foods (reviewed in [1]).

Experiments using ligated intestinal loops of animals and purified botulinum toxins showed that the upper small intestine was found to be the most important site for absorption of these toxins (reviewed in $[3,8]$ ). Further in vivo experiments using recent imaging technology under physiological conditions (no ligation) will provide a more detailed understanding of the sites of the absorption of botulinum toxin in the intestine. After passing through the intestinal epithelium, the toxins first appear in the lymph and then in the blood (reviewed in $[3,8]$ ). Botulinum toxin can also be absorbed from various mucous membranes, such as 
the mucous membranes of buccal cavity [53], stomach [54], and respiratory systems [55].

3.2. NAP Enhance the Oral Toxicity of BoNT by Their Protective Effects. The BoNT alone isolated from BoNT complexes was found to be only slightly toxic to mice when administrated orally. Its oral toxicity increases with the incremental association of the BoNT with the NAP (for a review, see [3]). Among six toxin serotypes (A to F), the type B (strain Lamanna) shows the most marked tendency, 16S toxin is about 1000 times more potent than $12 \mathrm{~S}$ toxin, and $12 \mathrm{~S}$ toxin is about 20 times more potent than BoNT in mice experiments [3]. Although the overall mechanism leading to the higher efficacy of the BoNT complexes is not fully understood, Sakaguchi's group has documented that their greater efficacy is due to the protective effect toward BoNT of the NAP from the low $\mathrm{pH}$ and proteases in the digestive tract [3]. Sakaguchi's group also reported that BoNT complexes do not dissociate in the digestive tract, including the duodenum in spite of the $\mathrm{pH}$ being around 7.0 [56], and the whole toxin complexes seem to be absorbed from the intestine into the lymphatics in rat ligated duodenum loop assay [9]; then molecular dissociation occurs immediately after BoNT complexes are absorbed into the lymphatics [9] (reviewed in [3]).

It was also reported that NAP plays a role in enhancing the neurotoxicity of BoNT, by a different mechanism from its protective effect against the external environment [57-60].

\subsection{Transcytosis of BoNT across the Intestinal Epithelial Cells.} In vitro experiments showed that BoNT/A and BoNT/B bind to polarized human intestinal epithelial cell lines (Caco-2 and T84) and undergo a transcytosis pathway from the apical to basolateral side [61-65]. The $\mathrm{H}$ chain of the BoNT is involved in binding and transcytosis in the intestinal cells $[62,64,66]$, and gangliosides (GD1b and GT1b series) and SV2 (or SV2 related protein) on the intestinal cell surface are possibly involved in this process [64]. It was also reported that an intestinal crypt like cell line derived from mouse (m-ICc12), which expresses a higher amount of SV2C (or SV2C related protein) than Caco-2, showed a higher level of binding and passage of BoNT/A than Caco-2 [64]. Although the BoNT transcytosis pathway has not yet been clearly defined, a recent study found that the BoNT/A C-terminal part of $\mathrm{H}$ chain $(\mathrm{Hc})$ enters preferentially via a Cdc42dependent and clathrin-independent pathway in Caco-2 and $\mathrm{m}-\mathrm{ICc} 12$ and reaches an early endosomal compartment [66].

3.4. HA Proteins Tether the BoNT to the Microvili of the Intestinal Epithelium. Several experiments were conducted to investigate the interaction of BoNT complexes with the intestinal epithelium. Experiments using ligated intestinal loops of guinea pigs and type $\mathrm{C}$ toxins (BoNT, $12 \mathrm{~S}$ toxin and $16 \mathrm{~S}$ toxin) showed that only $16 \mathrm{~S}$ toxin has a potent and selective binding activity to the microvilli of the upper small intestine via sialic acid residues in cell surface glycoconjugates [67]. This finding suggests the important role of the HA component in intestinal absorption of BoNT. Subsequently, it was shown that type C $16 \mathrm{~S}$ toxin, but not $12 \mathrm{~S}$ toxin or BoNT, binds and enters HT-29 cells (a human intestinal epithelial cell line) via the cell surface sialic acid containing O-liked glycoproteins [68]. When internalized into HT-29 cells, the type C16S toxin appears to be separated to the BoNT and NAP in endosome and the BoNT can further transferred to the Golgi apparatus [69]. In vivo, a substantial amount of ingested progenitor toxins seem to exist in their undissociated forms, at least until the BoNT complexes reach the luminal side of the intestine (see Section 3.2). These data led us to speculate that the HA in BoNT complex may play a role in the enhancement of BoNT absorption from the luminal surface of the intestinal epithelium to the circulating fluids, probably via transcytosis. Indeed, it was recently reported that HA1 of the type D $16 \mathrm{~S}$ toxin may play a critical role in facilitating the passage of BoNT through Caco2 monolayers [70]. However, it was shown that NAP does not facilitate the transcytosis of BoNT in T84, Caco-2, and Calu-3 (a human pulmonary cell line) (type A $[55,62,64]$ type B [62]). At present, both of these studies, which propose directly opposite ideas, were done using mainly in vitro cell lines and these systems are not always physiologically relevant. Multilateral approaches using such in situ intestinal epithelium of the susceptible species will thus be necessary to role out the function of NAP in the transcytosis of BoNT.

Since it was demonstrated that type C HA of the BoNT complex has a binding activity to the carbohydrates on the intestinal epithelial cell surfaces, additional studies have been carried out to characterize the binding property of other types of BoNT complexes. In type A, HA-positive BoNT complexes (mixture of $16 \mathrm{~S}$ toxin and 19S toxin) but not $12 \mathrm{~S}$ toxin show binding activity toward intestinal epithelial cells and erythrocytes, as in the case of type C BoNT complexes; whereas, the type A $16 \mathrm{~S}$ toxin recognizes galactose residues instead of sialic acid residues in the cell surface glycoconjugates of these cells [71]. In agreement with this observation, in erythrocytes, the Gal $\beta 1$-4GlcNAc moiety in the cell surface glycoconjugates has been determined as a major ligand for type A HA-positive BoNT complexes and HA1 (native HA1 exists in the culture medium) [72]. In the human intestinal cell line, Intestine-407, the Gal $\beta 1$ 4GlcNAc (N-acetyllactosamine) moiety in the cell surface oligosaccarides has been determined as a major ligand for type A HA-positive BoNT complexes [73].

Studies using recombinant HA proteins showed that HA1 and HA3b have carbohydrate binding activities with different specificities $[71,74,75]$. In type $C$, the HA1 recognizes NeuAc $\alpha 2-3$ Gal $\beta 1$ - and Gal $\beta 1$-4 GlcNAc $\beta 1$-; whereas, HA3b (and its precursor form, HA3) recognizes NeuAc $\alpha 2-3$ Gal $\beta 1$ [74]. Type C HAl has been shown to have two $\beta$-trefoil domains and bears similarities with the plant lectin ricin Bchain in its amino acid sequence [76] and crystal structure [49]. It has been demonstrated that each of these two $\beta$ trefoil domains (site I and site II) possesses a carbohydrate binding activity by viewing their X-ray crystal structures in complex with their carbohydrate ligands and point mutation analysis [50]. The site I $\beta$-trefoil domain bounds wide variety of sugars, such as $\mathrm{N}$-acetylneuraminic acid, $\mathrm{N}$ acetylgalactosamine, and galactose, while site II $\beta$-trefoil domain, which is located on the C-terminal side of site I, 


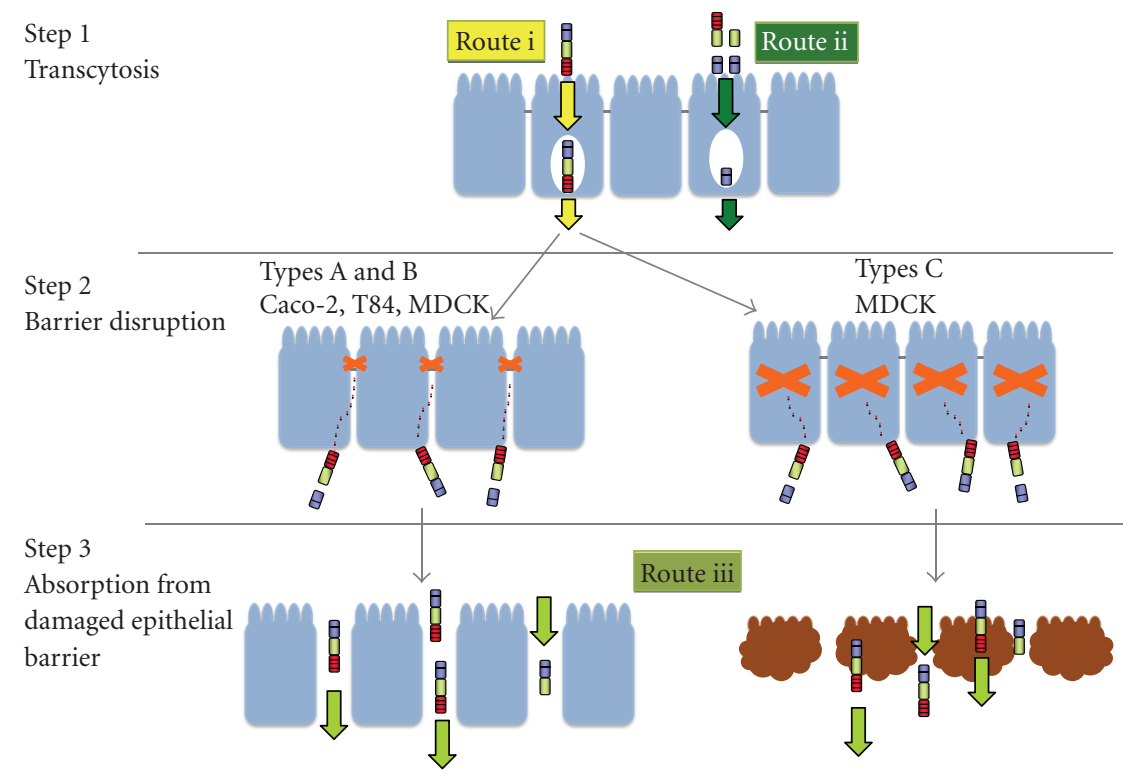

Figure 3: Model for penetration of BoNT complexes through the intestinal epithelial barrier. Step 1: HA of the BoNT complex mediates binding and transcytosis of a small amount of luminally located BoNT complexes across the epithelium without disrupting the epithelial barrier (Arrows in yellow, "route i") [65]. H chain of BoNT also mediates binding and transcytosis of BoNT (Arrows in green, "route ii") [61-66]. Step 2: HA which has translocated on the basolateral surface disrupts the epithelial barrier. Types A and B HA proteins disrupt the paracellular barrier of without causing cytotoxic effects in the epithelial cells of their susceptible hosts [65, 77]. Type C HA proteins possibly evoke cytotoxic-barrier disrupting activity in the epithelial cells of susceptible animals [77]. Step 3: A large amount of the BoNT complexes and BoNT accumulates in the serosal side by passing from the damaged epithelial barrier (Arrows in light green, "route iii") [65].

bounds only galactose and the binding avidity seems to be lower than that of site I. Regarding HA3, a binding site for N-acetylneuraminic acid within type C HA3 has been determined by X-ray crystallography and point mutation analysis [52]. What is the reason for the existence of multiple carbohydrate-binding sites with different specificities in the HA-positive BoNT complexes? Further studies are needed to clarify whether all of these three carbohydrate-binding sites are accessible in the complex form, whether, and how these carbohydrate-binding activities contribute to the passage of BoNT across the intestinal epithelial barrier.

3.5. HA Proteins Have Novel Activities That Disrupt the Epithelial Barrier Function. In 2008, we found a novel effect of the HA component; the type B HA disrupts the paracellular barrier of the intestinal epithelium and facilitates the transepithelial delivery of BoNT and other macromolecules both in vitro (human intestinal cell lines) and in vivo (mouse intestinal loop assay) [65]. On the other hand, this finding seems not to be in harmony with those reported by Sakaguchi who found that NAP does not enhance the rate of absorption of BoNT (in terms of antigenicity) in their in vivo rat intestinal loop assay [3]. The discrepancy may be due to species tropism in the action of HA; type B HA disrupts the paracellular barrier of the mouse, but not rat epithelium in the intestinal loop assay (manuscript in preparation).

We also found that type A HA proteins have a similar disrupting activity with a greater potency than type B HA proteins in Caco-2, T84, and MDCK I (canine kidney epithelial cell line) [77]. In contrast, type C HA proteins in the toxin complex (up to $300 \mathrm{nM}$ ) have no detectable effect on the paracellular barrier in these human cell lines [77]. These results indicate the correlation of the species tropism of HA action with the epidemiology of food-borne botulism. Therefore, HA action on the intestinal epithelial barrier may at least partially govern the susceptibility of different species to the toxicity of orally ingested BoNT complexes, and this may be an important factor in the pathogenesis of foodborne botulism.

In addition, we found that type C HA has a barrier disrupting activity and potent cytotoxicity in nonhuman originated certain epithelial cell lines (MDCK I, ACL-15, and RCN-9 derived from rat colon carcinomas) [77]. These data raise the possibility that type $\mathrm{C} \mathrm{HA}$ induces damage in the intestinal epithelium of susceptible animals, which could allow the toxin complex unrestricted influx into the systemic circulation. However, this evidence about the epithelial barrier disruption provoked by type $\mathrm{C}$ HA proteins is limited to in vitro cell lines. Further in vivo studies using susceptible animals will provide a better understanding of the pathological roles of this cytotoxic activity.

3.6. Possible HA-Mediated Three-Step Mechanism for the Intestinal Absorption of BoNT Complex. Our studies indicated that the type $\mathrm{A} \sim \mathrm{C}$ HA proteins present in the $16 \mathrm{~S}$ toxin act on undefined host molecules, mainly on the basolateral surface, and these induce the loss of the epithelial paracellular barrier without causing cytotoxicity (Types A and B HA) or the loss of general epithelial barrier function accompanied by cell damage (Types C HA), which facilitates the efficient transepithelial-absorption of luminal macromolecules including BoNT $[65,77]$. Taking into account these findings 
and other group's findings, we propose a possible threestep mechanism by which the toxin traverses the intestinal epithelial barrier (Figure 3): Step 1, transport of a small amount of luminally located BoNT complexes [65] or BoNT alone [61-66] across the epithelium without disrupting the epithelial barrier via transcytosis; Step 2, disruption of the epithelial barrier by the HA moiety of the $16 \mathrm{~S}$ toxin that has translocated to the basolateral surface $[65,77]$; and Step 3, accumulation of a large amount of BoNT complexes (and BoNT) in the serosal side by passing through the damaged epithelial barrier [65]. As previously mentioned, the in vivo relevance of these steps is at present obscure and necessary to be confirmed experimentally.

\section{Conclusions and Perspectives}

In these few years, significant progress has been made in our understanding of the interaction of botulinum toxin with the intestinal epithelial barrier. It has become clear that the type $\mathrm{A}, \mathrm{B}$, and $\mathrm{C}$ HA proteins in BoNT complexes possess a potent ability to disrupts epithelial barrier function and have distinct features in their modes of action. These findings provide an awareness of botulinum HA proteins as pathogenic factors that breach the host defense by direct interaction with the host epithelium, which is presumably linked to the intestinal transepithelial delivery of BoNT in food-borne botulism of susceptible species. However, the molecular mechanisms by which each type of $\mathrm{A} \sim \mathrm{C} \mathrm{HA}$ proteins disrupt intestinal epithelial barriers remain unclear. Similarly, it is unclear whether the lectin activities of HA 3 and HA1 are involved in these epithelial barrier disrupting activities. Overall, there are at least three possible routes taken by botulinum toxin to penetrate the gut epithelium; route i, HA-mediated transcytosis ("Step 1" described in chapter III-6, chapter III-4) [65], route ii, H chain-mediated transcytosis of BoNT alone ("Step 1" described in chapter III-6, chapter III-3) [61-66], and route iii, the route passing from the damaged epithelial barrier caused by HA actions ("Step 3" described in chapter III-6, chapter III-5) [65]. Which route(s) are taken and to what extent are they involved in the absorption of the toxin in food-borne botulism? To answer these questions, further studies to elucidate the molecular interactions that occur between the botulinum toxin (complex) and the intestinal epithelial barrier and in vivo validation using appropriate species are necessary. These studies not only will provide an important insight into the molecular mechanisms behind the development of foodborne botulism but also may lead to unique and powerful opportunities to understand the complicated mechanisms for the maintenance and regulation of the epithelial barrier system. Moreover, the mechanisms by which botulinum toxin traverses the intestinal epithelial barrier could be exploited to allow delivery of drugs across the epithelium.

\section{A List of Abbreviations}

BoNT: Botulinum neurotoxin

HA: Hemagglutinin

NTX: Botulinum neurotoxin
NTNH: Nontoxic nonhemagglutinin

NTNHA: Nontoxic nonhemagglutinin

NAP: $\quad$ Nontoxic neurotoxin-associated protein

TC: $\quad$ Toxin complex.

\section{Acknowledgments}

The studies described in this review were partly supported by grants from the Ministry of Education, Culture, Sports, Science, and Technology of Japan (Y. Fujinaga, T. Matsumura), from the Kato Memorial Bioscience Foundation (Y. Fujinaga), and from Suzuken Memorial Foundation (Y. Fujinaga).

\section{References}

[1] S. S. Arnon, R. Schechter, T. V. Inglesby, et al., "Botulinum toxin as a biological weapon: medical and public health management," Journal of the American Medical Association, vol. 285, no. 8, pp. 1059-1070, 2001.

[2] G. Schiavo, M. Matteoli, and C. Montecucco, "Neurotoxins affecting neuroexocytosis," Physiological Reviews, vol. 80, no. 2, pp. 717-766, 2000.

[3] G. Sakaguchi, "Clostridium botulinum toxins," Pharmacology \& Therapeutics, vol. 19, no. 2, pp. 165-194, 1982.

[4] N. P. Minton, "Molecular genetics of clostridial neurotoxins," Current Topics in Microbiology and Immunology, vol. 195, pp. 161-194, 1995.

[5] M. D. Collins and A. K. East, "Phylogeny and taxonomy of the food-borne pathogen Clostridium botulinum and its neurotoxins," Journal of Applied Microbiology, vol. 84, no. 1, pp. 5-17, 1998.

[6] K. Oguma, K. Inoue, Y. Fujinaga, et al., "Structure and function of Clostridium botulinum progenitor toxin," Journal of Toxicology-Toxin Reviews, vol. 18, no. 1, pp. 17-34, 1999.

[7] B. Poulain, M. R. Popoff, and J. Molgo, "How do the Botulinum Neurotoxins block neurotransmitter release: from botulinum to the molecular mechanism of action," The Botulinum Journal, vol. 1, no. 1, pp. 14-87, 2008.

[8] P. F. Bonventre, "Absorption of botulinal toxin from the gastrointestinal tract," Reviews of Infectious Diseases, vol. 1, no. 4, pp. 663-667, 1979.

[9] S. Sugii, I. Ohishi, and G. Sakaguchi, "Intestinal absorption of botulinum toxins of different molecular sizes in rats," Infection and Immunity, vol. 17, no. 3, pp. 491-496, 1977.

[10] T. Binz and A. Rummel, "Cell entry strategy of clostridial neurotoxins," Journal of Neurochemistry, vol. 109, no. 6, pp. 1584-1595, 2009.

[11] T. J. Smith, J. Lou, I. N. Geren, et al., "Sequence variation within botulinum neurotoxin serotypes impacts antibody binding and neutralization," Infection and Immunity, vol. 73, no. 9, pp. 5450-5457, 2005.

[12] J. W. Arndt, M. J. Jacobson, E. E. Abola, et al., "A structural perspective of the sequence variability within Botulinum Neurotoxin subtypes A1-A4," Journal of Molecular Biology, vol. 362, no. 4, pp. 733-742, 2006.

[13] K. K. Hill, T. J. Smith, C. H. Helma, et al., "Genetic diversity among botulinum neurotoxin-producing clostridial strains," Journal of Bacteriology, vol. 189, no. 3, pp. 818-832, 2007.

[14] T. J. Smith, K. K. Hill, B. T. Foley, et al., "Analysis of the neurotoxin complex genes in Clostridium botulinum A1-A4 and B1 strains: BoNT/A3, /Ba4 and /B1 clusters are located within plasmids," PLoS One, vol. 2, no. 12, p. e1271, 2007. 
[15] A. T. Carter, C. J. Paul, D. R. Mason, et al., "Independent evolution of neurotoxin and flagellar genetic loci in proteolytic Clostridium botulinum," BMC Genomics, vol. 10, article 115, 2009.

[16] K. Inoue, Y. Fujinaga, T. Watanabe, et al., "Molecular composition of Clostridium botulinum type A progenitor toxins," Infection and Immunity, vol. 64, no. 5, pp. 1589-1594, 1996.

[17] M. Dong, F. Yeh, W. H. Tepp, et al., "SV2 is the protein receptor for botulinum neurotoxin A," Science, vol. 312, no. 5773, pp. 592-596, 2006.

[18] S. Mahrhold, A. Rummel, H. Bigalke, B. Davletov, and T. Binz, "The synaptic vesicle protein $2 \mathrm{C}$ mediates the uptake of botulinum neurotoxin A into phrenic nerves," FEBS Letters, vol. 580, no. 8, pp. 2011-2014, 2006.

[19] M. Dong, H. Liu, W. H. Tepp, E. A. Johnson, R. Janz, and E. R. Chapman, "Glycosylated SV2A and SV2B mediate the entry of botulinum neurotoxin E into neurons," Molecular Biology of the Cell, vol. 19, no. 12, pp. 5226-5237, 2008.

[20] A. Rummel, K. Hafner, S. Mahrhold, et al., "Botulinum neurotoxins $\mathrm{C}, \mathrm{E}$ and $\mathrm{F}$ bind gangliosides via a conserved binding site prior to stimulation-dependent uptake with botulinum neurotoxin $\mathrm{F}$ utilising the three isoforms of SV2 as second receptor," Journal of Neurochemistry, vol. 110, no. 6, pp. 1942-1954, 2009.

[21] T.-I. Nishiki, Y. Kamata, Y. Nemoto, et al., "Identification of protein receptor for Clostridium botulinum type B neurotoxin in rat brain synaptosomes," Journal of Biological Chemistry, vol. 269, no. 14, pp. 10498-10503, 1994.

[22] T.-I. Nishiki, Y. Tokuyama, Y. Kamata, et al., "The highaffinity binding of Clostridium botulinum type B neurotoxin to synaptotagmin II associated with gangliosides GT1b/GD1a," FEBS Letters, vol. 378, no. 3, pp. 253-257, 1996.

[23] T.-I. Nishiki, Y. Tokuyama, Y. Kamata, et al., "Binding of botulinum type B neurotoxin to Chinese hamster ovary cells transfected with rat synaptotagmin II cDNA," Neuroscience Letters, vol. 208, no. 2, pp. 105-108, 1996.

[24] M. Dong, D. A. Richards, M. C. Goodnough, W. H. Tepp, E. A. Johnson, and E. R. Chapman, "Synaptotagmins I and II mediate entry of botulinum neurotoxin B into cells," Journal of Cell Biology, vol. 162, no. 7, pp. 1293-1303, 2003.

[25] A. Rummel, T. Karnath, T. Henke, H. Bigalke, and T. Binz, "Synaptotagmins I and II act as nerve cell receptors for botulinum neurotoxin G," Journal of Biological Chemistry, vol. 279, no. 29, pp. 30865-30870, 2004.

[26] M. Dong, W. H. Tepp, H. Liu, E. A. Johnson, and E. R. Chapman, "Mechanism of botulinum neurotoxin B and G entry into hippocampal neurons," Journal of Cell Biology, vol. 179, no. 7, pp. 1511-1522, 2007.

[27] A. Rummel, T. Eichner, T. Weil, et al., "Identification of the protein receptor binding site of botulinum neurotoxins $\mathrm{B}$ and G proves the double-receptor concept," Proceedings of the National Academy of Sciences of the United States of America, vol. 104, no. 1, pp. 359-364, 2007.

[28] K. Tsukamoto, T. Kohda, M. Mukamoto, et al., "Binding of Clostridium botulinum type $\mathrm{C}$ and $\mathrm{D}$ neurotoxins to ganglioside and phospholipid: novel insights into the receptor for clostridial neurotoxins," Journal of Biological Chemistry, vol. 280, no. 42, pp. 35164-35171, 2005.

[29] L. K. Koriazova and M. Montal, "Translocation of botulinum neurotoxin light chain protease through the heavy chain channel," Nature Structural Biology, vol. 10, no. 1, pp. 13-18, 2003.
[30] A. Fischer and M. Montal, "Single molecule detection of intermediates during botulinum neurotoxin translocation across membranes," Proceedings of the National Academy of Sciences of the United States of America, vol. 104, no. 25, pp. 10447-10452, 2007.

[31] A. Fischer, D. J. Mushrush, D. B. Lacy, et al., "Botulinum neurotoxin devoid of receptor binding domain translocates active protease," PLoS Pathogens, vol. 4, no. 12, Article ID e1000245, 2008.

[32] D. B. Lacy, W. Tepp, A. C. Cohen, B. R. DasGupta, and R. C. Stevens, "Crystal structure of botulinum neurotoxin type A and implications for toxicity," Nature Structural Biology, vol. 5, no. 10, pp. 898-902, 1998.

[33] R. Agarwal, S. Eswaramoorthy, D. Kumaran, T. Binz, and S. Swaminathan, "Structural analysis of botulinum neurotoxin type E catalytic domain and its mutant Glu212 $\rightarrow$ Gln reveals the pivotal role of the Glu212 carboxylate in the catalytic pathway," Biochemistry, vol. 43, no. 21, pp. 6637-6644, 2004.

[34] R. Agarwal, T. Binz, and S. Swaminathan, "Structural analysis of botulinum neurotoxin serotype F light chain: implications on substrate binding and inhibitor design," Biochemistry, vol. 44, no. 35, pp. 11758-11765, 2005.

[35] J. W. Arndt, W. Yu, F. Bi, et al., "Crystal structure of botulinum neurotoxin type $\mathrm{G}$ light chain: serotype divergence in substrate recognition," Biochemistry, vol. 44, no. 28, pp. 9574-9580, 2005.

[36] J. W. Arndt, Q. Chai, T. Christian, and R. C. Stevens, "Structure of botulinum neurotoxin type D light chain at 1.65 A resolution: repercussions for VAMP-2 substrate specificity," Biochemistry, vol. 45, no. 10, pp. 3255-3262, 2006.

[37] D. Kumaran, S. Eswaramoorthy, W. Furey, J. Navaza, M. Sax, and S. Swaminathan, "Domain organization in Clostridium botulinum neurotoxin type $\mathrm{E}$ is unique: its implication in faster translocation," Journal of Molecular Biology, vol. 386, no. 1, pp. 233-245, 2009.

[38] M. A. Breidenbach and A. T. Brunger, "Substrate recognition strategy for butulinum neurotoxin serotype A," Nature, vol. 432, no. 7019, pp. 925-929, 2004.

[39] B. Segelke, M. Knapp, S. Kadkhodayan, R. Balhorn, and B. Rupp, "Crystal structure of Clostridium botulinum neurotoxin protease in a product-bound state: evidence for noncanonical zinc protease activity," Proceedings of the National Academy of Sciences of the United States of America, vol. 101, no. 18, pp. 6888-6893, 2004.

[40] D. Kumaran, R. Rawat, S. A. Ahmed, et al., "Substrate binding mode and its implication on drug design for botulinum neurotoxin A," PLoS Pathogens, vol. 4, no. 9, Article ID e1000165, 2008.

[41] S. Swaminathan and S. Eswaramoorthy, "Structural analysis of the catalytic and binding sites of Clostridium botulinum neurotoxin B," Nature Structural Biology, vol. 7, no. 8, pp. 693699, 2000.

[42] Q. Chai, J. W. Arndt, M. Dong, et al., "Structural basis of cell surface receptor recognition by botulinum neurotoxin B," Nature, vol. 444, no. 7122, pp. 1096-1100, 2006.

[43] R. Jin, A. Rummel, T. Binz, and A. T. Brunger, "Botulinum neurotoxin $B$ recognizes its protein receptor with high affinity and specificity," Nature, vol. 444, no. 7122, pp. 1092-1095, 2006.

[44] P. Stenmark, J. Dupuy, A. Imamura, et al., "Crystal structure of botulinum neurotoxin type A in complex with the cell surface co-receptor GT1b-insight into the toxin-neuron interaction," PLoS Pathogens, vol. 4, no. 8, Article ID e1000129, 2008. 
[45] K. Miyata, T. Yoneyama, T. Suzuki, et al., "Expression and stability of the nontoxic component of the botulinum toxin complex," Biochemical and Biophysical Research Communications, vol. 384, no. 1, pp. 126-130, 2009.

[46] K. Hasegawa, T. Watanabe, T. Suzuki, et al., "A novel subunit structure of Clostridium botulinum serotype D toxin complex with three extended arms," The Journal of Biological Chemistry, vol. 282, no. 34, pp. 24777-24783, 2007.

[47] S. Mutoh, H. Kouguchi, Y. Sagane, et al., "Complete subunit structure of the Clostridium botulinum type D toxin complex via intermediate assembly with nontoxic components," Biochemistry, vol. 42, no. 37, pp. 10991-10997, 2003.

[48] T. Suzuki, T. Watanabe, S. Mutoh, et al., "Characterization of the interaction between subunits of the botulinum toxin complex produced by serotype D through tryptic susceptibility of the isolated components and complex forms," Microbiology, vol. 151, part 5, pp. 1475-1483, 2005.

[49] K. Inoue, M. Sobhany, T. R. Transue, et al., "Structural analysis by X-ray crystallography and calorimetry of a haemagglutinin component (HA1) of the progenitor toxin from Clostridium botulinum," Microbiology, vol. 149, part 12, pp. 3361-3370, 2003.

[50] T. Nakamura, T. Tonozuka, A. Ide, T. Yuzawa, K. Oguma, and A. Nishikawa, "Sugar-binding sites of the HA1 subcomponent of Clostridium botulinum type C progenitor toxin," Journal of Molecular Biology, vol. 376, no. 3, pp. 854-867, 2008.

[51] J. W. Arndt, J. Gu, L. Jaroszewski, et al., "The structure of the neurotoxin-associated protein HA33/A from Clostridium botulinum suggests a reoccurring $\beta$-trefoil fold in the progenitor toxin complex," Journal of Molecular Biology, vol. 346, no. 4, pp. 1083-1093, 2005.

[52] T. Nakamura, M. Kotani, T. Tonozuka, A. Ide, K. Oguma, and A. Nishikawa, "Crystal structure of the HA3 subcomponent of Clostridium botulinum type C progenitor toxin," Journal of Molecular Biology, vol. 385, no. 4, pp. 1193-1206, 2009.

[53] C. Lamanna, R. A. Hillowalla, and C. C. Alling, "Buccal exposure to botulinal toxin," Journal of Infectious Diseases, vol. 117, no. 4, pp. 327-331, 1967.

[54] A. B. Maksymowych, M. Reinhard, C. J. Malizio, M. C. Goodnough, E. A. Johnson, and L. L. Simpson, "Pure botulinum neurotoxin is absorbed from the stomach and small intestine and produces peripheral neuromuscular blockade," Infection and Immunity, vol. 67, no. 9, pp. 4708-4712, 1999.

[55] J.-B. Park and L. L. Simpson, "Inhalational poisoning by botulinum toxin and inhalation vaccination with its heavychain component," Infection and Immunity, vol. 71, no. 3, pp. 1147-1154, 2003.

[56] S. Sugii, I. Ohishi, and G. Sakaguchi, "Correlation between oral toxicity and in vitro stability of Clostridium botulinum type A and B toxins of different molecular sizes," Infection and Immunity, vol. 16, no. 3, pp. 910-914, 1977.

[57] S. Cai, H. K. Sarkar, and B. R. Singh, "Enhancement of the endopeptidase activity of botulinum neurotoxin by its associated proteins and dithiothreitol," Biochemistry, vol. 38, no. 21, pp. 6903-6910, 1999.

[58] S. K. Sharma and B. R. Singh, "Enhancement of the endopeptidase activity of purified botulinum neurotoxins A and E by an isolated component of the native neurotoxin associated proteins," Biochemistry, vol. 43, no. 16, pp. 4791-4798, 2004.

[59] Y. Zhou, S. Foss, P. Lindo, H. Sarkar, and B. R. Singh, "Hemagglutinin-33 of type A botulinum neurotoxin complex binds with synaptotagmin II," FEBS Journal, vol. 272, no. 11, pp. 2717-2726, 2005.
[60] R. V. Kukreja and B. R. Singh, "Comparative role of neurotoxin-associated proteins in the structural stability and endopeptidase activity of botulinum neurotoxin complex types A and E," Biochemistry, vol. 46, no. 49, pp. 14316-14324, 2007.

[61] A. B. Maksymowych and L. L. Simpson, "Binding and transcytosis of botulinum neurotoxin by polarized human colon carcinoma cells," Journal of Biological Chemistry, vol. 273, no. 34, pp. 21950-21957, 1998.

[62] A. B. Maksymowych and L. L. Simpson, "Structural features of the botulinum neurotoxin molecule that govern binding and transcytosis across polarized human intestinal epithelial cells," Journal of Pharmacology and Experimental Therapeutics, vol. 310, no. 2, pp. 633-641, 2004.

[63] C. R. Ahsan, G. Hajnoczky, A. B. Maksymowych, and L. L. Simpson, "Visualization of binding and transcytosis of botulinum toxin by human intestinal epithelial cells," Journal of Pharmacology and Experimental Therapeutics, vol. 315, no. 3, pp. 1028-1035, 2005.

[64] A. Couesnon, Y. Pereira, and M. R. Popoff, "Receptormediated transcytosis of botulinum neurotoxin A through intestinal cell monolayers," Cellular Microbiology, vol. 10, no. 2, pp. 375-387, 2008.

[65] T. Matsumura, Y. Jin, Y. Kabumoto, et al., "The HA proteins of botulinum toxin disrupt intestinal epithelial intercellular junctions to increase toxin absorption," Cellular Microbiology, vol. 10, no. 2, pp. 355-364, 2008.

[66] A. Couesnon, T. Shimizu, and M. R. Popoff, "Differential entry of botulinum neurotoxin A into neuronal and intestinal cells," Cellular Microbiology, vol. 11, no. 2, pp. 289-308, 2009.

[67] Y. Fujinaga, K. Inoue, S. Watanabe, et al., "The haemagglutinin of Clostridium botulinum type $\mathrm{C}$ progenitor toxin plays an essential role in binding of toxin to the epithelial cells of guinea pig small intestine, leading to the efficient absorption of the toxin," Microbiology, vol. 143, part 12, pp. 3841-3847, 1997.

[68] A. Nishikawa, N. Uotsu, H. Arimitsu, et al., "The receptor and transporter for internalization of Clostridium botulinum type C progenitor toxin into HT-29 cells," Biochemical and Biophysical Research Communications, vol. 319, no. 2, pp. 327333, 2004.

[69] N. Uotsu, A. Nishikawa, T. Watanabe, et al., "Cell internalization and traffic pathway of Clostridium botulinum type C neurotoxin in HT-29 cells," Biochimica et Biophysica Acta, vol. 1763, no. 1, pp. 120-128, 2006.

[70] K. Niwa, K. Koyama, S.-I. Inoue, et al., "Role of nontoxic components of serotype $\mathrm{D}$ botulinum toxin complex in permeation through a Caco-2 cell monolayer, a model for intestinal epithelium," FEMS Immunology and Medical Microbiology, vol. 49, no. 3, pp. 346-352, 2007.

[71] Y. Fujinaga, K. Inoue, T. Nomura, et al., "Identification and characterization of functional subunits of Clostridium botulinum type A progenitor toxin involved in binding to intestinal microvilli and erythrocytes," FEBS Letters, vol. 467, no. 2-3, pp. 179-183, 2000.

[72] K. Inoue, Y. Fujinaga, K. Honke, et al., "Clostridium botulinum type A haemagglutinin-positive progenitor toxin ( $\mathrm{HA}^{+}$-PTX) binds to oligosaccharides containing Gal beta1-4GlcNAc through one subcomponent of haemagglutinin (HA1)," Microbiology, vol. 147, part 4, pp. 811-819, 2001.

[73] S. Kojima, H. Eguchi, T. Ookawara, et al., "Clostridium botulinum type A progenitor toxin binds to Intestine-407 cells via N-acetyllactosamine moiety," Biochemical and Biophysical Research Communications, vol. 331, no. 2, pp. 571-576, 2005. 
[74] Y. Fujinaga, K. Inoue, S. Watarai, et al., "Molecular characterization of binding subcomponents of Clostridium botulinum type $\mathrm{C}$ progenitor toxin for intestinal epithelial cells and erythrocytes," Microbiology, vol. 150, pp. 1529-1538, 2004.

[75] H. Arimitsu, Y. Sakaguchi, J.-C. Lee, et al., "Molecular properties of each subcomponent in Clostridium botulinum type B haemagglutinin complex," Microbial Pathogenesis, vol. 45, no. 2, pp. 142-149, 2008.

[76] Y. Sagane, H. Kouguchi, T. Watanabe, et al., "Role of Cterminal region of HA-33 component of botulinum toxin in hemagglutination," Biochemical and Biophysical Research Communications, vol. 288, no. 3, pp. 650-657, 2001.

[77] Y. Jin, Y. Takegahara, Y. Sugawara, et al., "Disruption of the epithelial barrier by botulinum haemagglutinin (HA) proteins-differences in cell tropism and the mechanism of action between HA proteins of types A or B, and HA proteins of type C," Microbiology, vol. 155, part 1, pp. 35-45, 2009. 

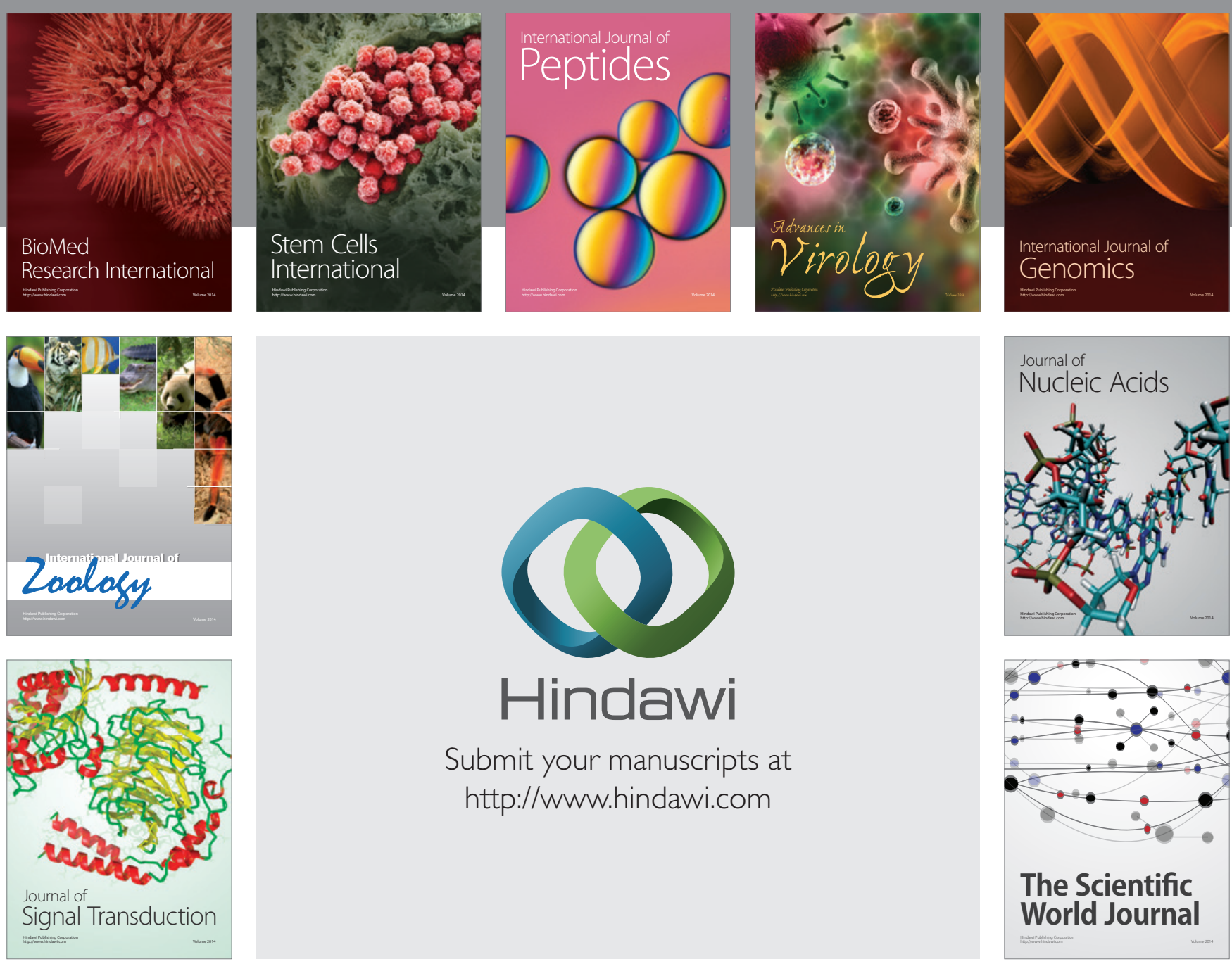

Submit your manuscripts at

http://www.hindawi.com
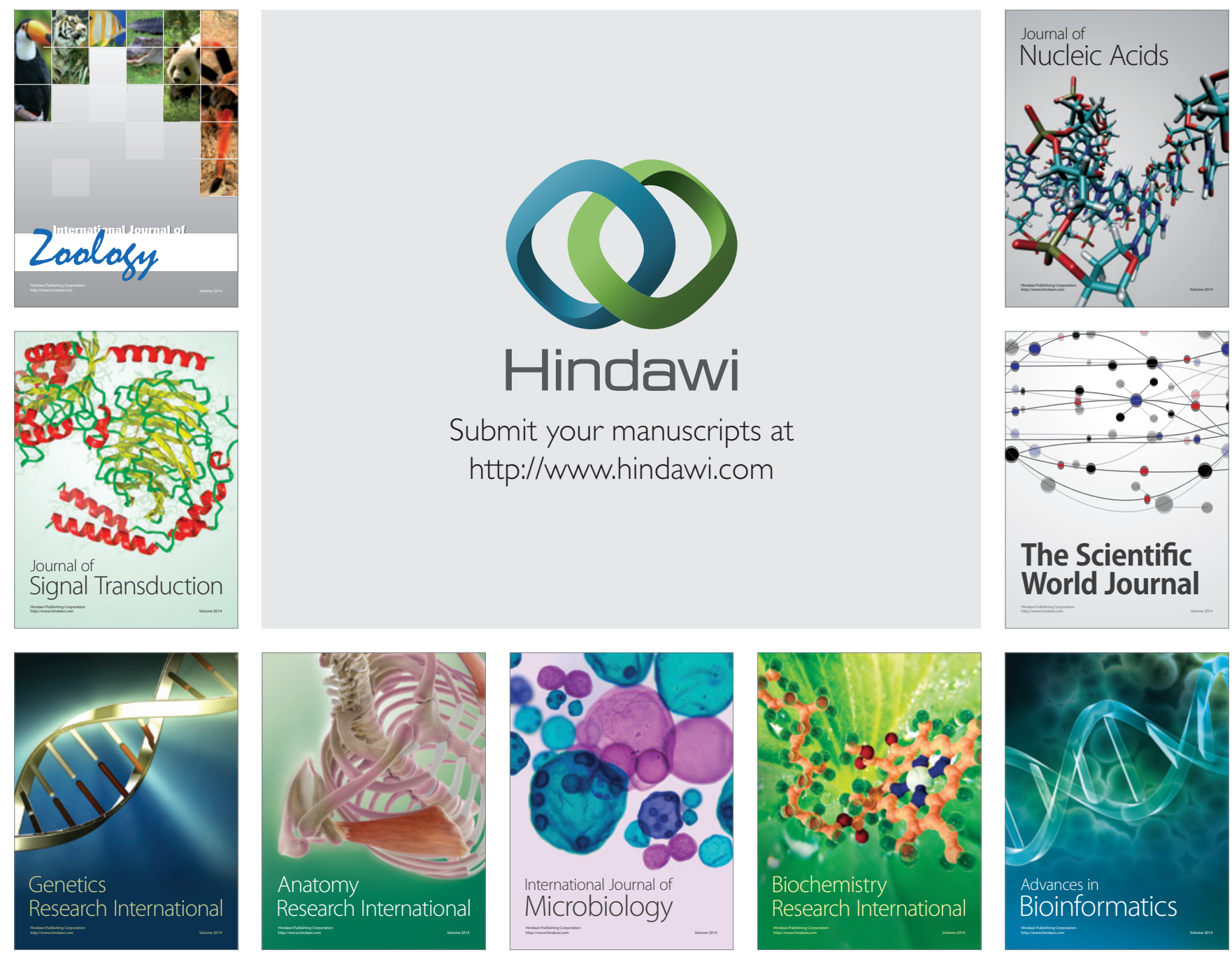

The Scientific World Journal
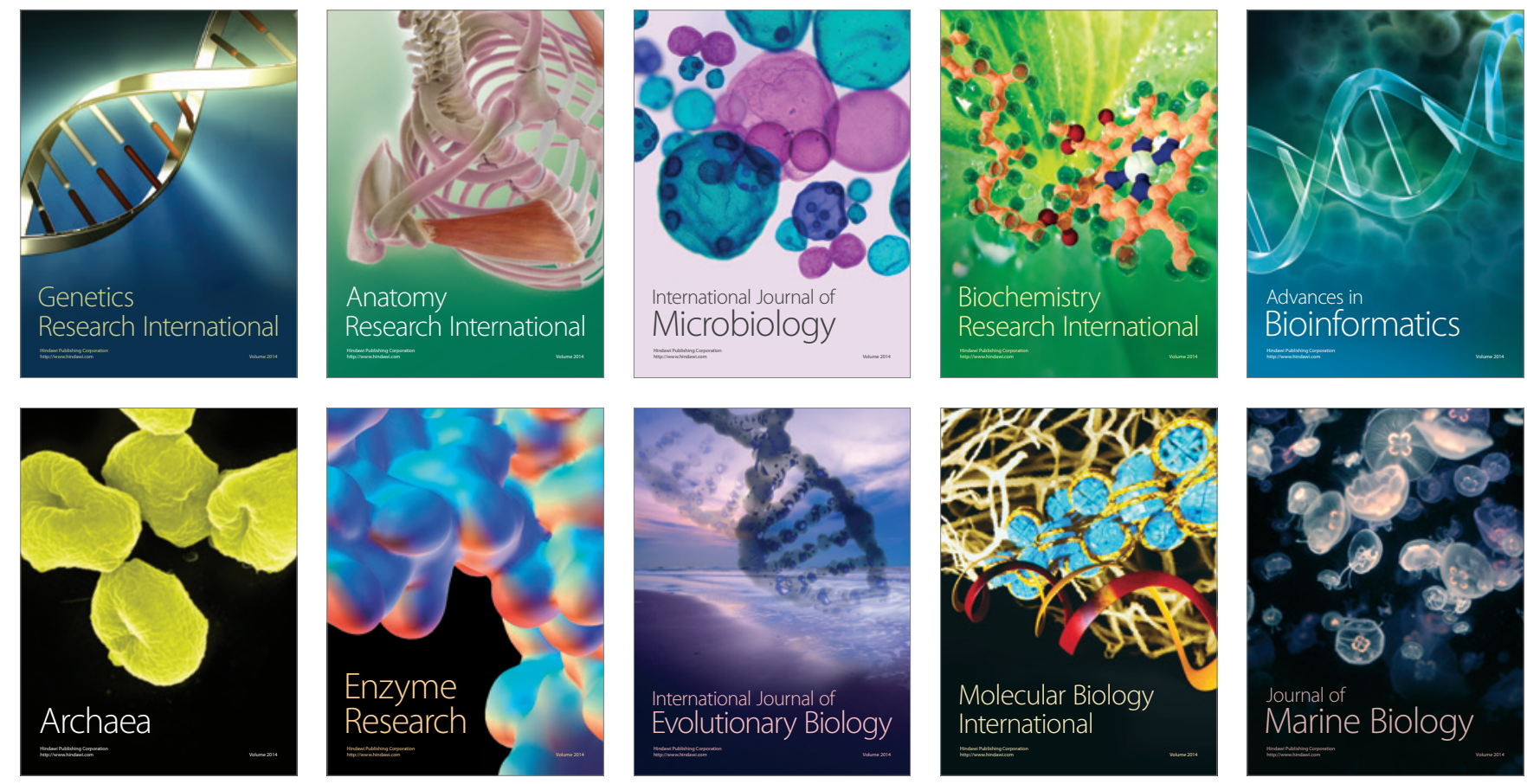